\title{
Takotsubo cardiomyopathy in the setting of multiple sclerosis: a multifaceted phenomenon with important implications
}

\author{
Kenan Yalta, ${ }^{1}$ Gokay Taylan, ${ }^{1}$ Tulin Yalta, ${ }^{2}$ Ertan Yetkın, ${ }^{3}$ \\ ${ }^{1}$ Cardiology Department, Trakya University, Edirne; ${ }^{2}$ Pathology Department, Trakya University, Edirne; ${ }^{3}$ Cardiology \\ Department, Liv Hospital, Istinye University, Istanbul, Turkey
}

\section{Dear Editor,}

Takotsubo cardiomyopathy (TTC) has been universally regarded as a unique form of reversible myocardial dysfunction associated with a variety of emotional and physical stressors [1-3]. In their recently published elegant article [1], Dell'Aquila et al. have reported an interesting case of TTC triggered by an exacerbation of relapsing-remitting multiple sclerosis (MS). However, we would like to comment on this interesting case and its particular implications.

First, TTC associated with physical stressors (including neurological diseases etc.) was previously suggested to have a significantly worse prognosis largely attributable to the severity of certain associated factors including adrenergic discharge, coronary microvascular dysfunction as well as systemic inflammatory response [2]. Notably, direct involvement of the cardiovascular

Correspondence: Kenan Yalta, Trakya University, Cardiology Department, Edirne, Turkey.

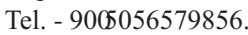

E-mail:kyalta@gmail.com, akenanyalta@trakya.edu.tr

Contributions: $\mathrm{KY} \square$ Design, conception, acquisition of literature data, analysis, interpretation, substantial writing, drafting, final revision and approval, affirming responsibility for the final content; GT Design, conception, acquisition of literature data, analysis, interpretation, writing, drafting, final revision and approval, affirming responsibility for the final content; TY $\square$ Design, conception, acquisition of literature data, analysis, interpretation, writing, drafting, final revision and approval, affirming responsibility for the final content; EY₫Design, conception, acquisition of literature data, analysis, interpretation, writing, drafting, final revision and approval, affirming responsibility for the final content.

Conflict of interest: The authors declare no conflict of interest.

Ethics approval and consent to participate: Not applicable.

Availability of data and materials: Not applicable.

Received for publication: 31 May 2020.

Accepted for publication: 19 June 2020.

${ }^{\circ}$ Copyright: the Author(s), 2020

Licensee PAGEPress, Italy

Monaldi Archives for Chest Disease 2020; 90:1420

doi: 10.4081/monaldi.2020.1420

This article is distributed under the terms of the Creative Commons Attribution Noncommercial License (by-nc 4.0) which permits any noncommercial use, distribution, and reproduction in any medium, provided the original author(s) and source are credited. centre in medulla oblongata generally serves as the fundamental trigger of TTC evolution in patients with a MS relapse [1]. Therefore, TTC in these patients might even have a worse clinical outcome due to the extreme and prolonged adrenergic discharge that usually outlasts the whole period of an emerging MS relapse. In the setting of TTC, severe adrenergic discharge has the potential to induce serious complications including malignant ventricular arrhythmias [2,3]. Moreover, acute coronary microvascular dysfunction (manifesting as a Coronary Slow Flow, CSF, pattern on invasive Coronary Angiogram, CAG) also associated with extreme adrenergic discharge might further aggravate the shortterm prognosis of TTC [2]. Accordingly, we wonder whether the patient [1] had any new-onset cardiac arrhythmias or significant CSF pattern which might be indicative of an existing substantial adrenergic discharge in the hospital setting.

Second, systemic inflammation might potentially contribute to the evolution as well as prognosis of TTC associated with physical stressors [2]. Accordingly, we wonder about serum levels of inflammation markers in the patient [1]. Importantly, quantification of such markers in patients with an MS relapse might enable further risk-stratification of the associated TTC episode [2]. Moreover, anti-inflammatory strategies used to treat MS relapses [1] might also improve the prognosis of associated TTC episode in this particular setting [2].

Third, TTC evolution in certain MS cases might exclusively be due to emotional triggers. It is well known that a portion of these cases might suffer emotional instability particularly during relapses, potentially warranting psychiatric counselling. Therefore, TTC evolution in MS relapses is quite possible even in the absence of specific lesions in medulla oblongata. Furthermore, MS patients in complete remission, yet with residual neurological deficit, might also suffer emotionally-triggered TTC episodes due to their potentially lower threshold for emotional surges. Accordingly, we wonder about psychiatric status of the patient during her relapse and remission periods.

Finally, due to the well-known risk of future TTC recurrences in patients with neurological and psychiatric diseases, every effort should be made to prevent future MS relapses and hence potential TTC recurrences in this particular case [1]. Therefore, we hold the opinion that the patient might also need an efficient long-term MS prophylaxis for TTC prevention even if clinical assessment of her current radiological and neurological status might not strictly warrant such neuroprophylactic strategies at this stage.

In conclusion, TTC in the setting of MS might be considered as a multifaceted and complex phenomenon with a variety of underlying mechanisms that mostly, but not exclusively, signify the acute impairment of cardiovascular centre in the brainstem. Moreover, TTC in this particular setting might also have important prognostic and therapeutic implications that deserve further attention in clinical practice. 
2. Yalta K, Yalta T. Physically triggered takotsubo cardiomyopathy has a worse prognosis: Potential roles of systemic inflammation and coronary slow flow phenomenon. Int J Cardiol 2017;242:31-32.

1. Dell'Aquila A, Sciatti E, Vizzardi E, et al. The brain-heart connection: a multiple sclerosis relapse presenting as Takotsubo Syndrome. A case report and literature review. Monaldi Arch Chest Dis 2020;90. doi: 10.4081/monaldi.2020.1153.

3. Yalta K, Yilmaztepe M, Zorkun C. Left Ventricular Dysfunction in the Setting of Takotsubo Cardiomyopathy: A Review of Clinical Patterns and Practical Implications. Card Fail Rev 2018;4:14-20. 